\title{
Nonlinear Computational Homogenization Method for the Evaluation of Eddy Currents in Soft Magnetic Composites
}

\author{
Innocent Niyonzima $^{1}$, Ruth Vazquez Sabariego ${ }^{1}$, Patrick Dular ${ }^{1,2}$ and Christophe Geuzaine ${ }^{1}$ \\ ${ }^{1}$ Dept. of Electrical Engineering and Computer Science (ACE), University of Liège, Belgium \\ ${ }^{2}$ Fonds de la Recherche Scientifique, F.R.S.-FNRS, Belgium.
}

\begin{abstract}
In this paper, a heterogeneous multiscale method (HMM) based technique is applied to model the behaviour of electromagnetic fields in soft magnetic composites (SMC). Two problems are derived from the two-scale homogenization theory: a macroscale problem that captures the slow variations of the overall solution, and many mesoscale problems that allow determining the constitutive laws at the macroscale. As application, an SMC core is considered.
\end{abstract}

Index Terms-Composite materials, multiscale homogenization, finite element methods, eddy currents, magnetic hysteresis.

\section{INTRODUCTION}

The use of the soft magnetic composites (SMC) in electric devices has recently increased. These materials, made from a metallic powder compacted with a dielectric binder, are a good alternative to laminated ferromagnetic structures as their granular mesoscale structure allows to significantly reduce the eddy current losses. Furthermore unlike the laminated ferromagnetic structures, SMC exhibit isotropic magnetic properties what makes them good candidates for manufacturing machines with 3-D flux paths.

The use of classical numerical methods such as the finite element (FE) method to accurately study the behaviour of $\mathrm{SMC}$ is computationally very expensive. Indeed a very fine mesh is required to capture the fine scale variations, i.e. the variations at the level of metallic grains, whence the need of multiscale methods and homogenization methods. The first homogenization approach used to analytically characterize homogenized properties of composites materials is based on mixing rules [1], [2]. Using this method, it is possible to determine equivalent properties with little information on the microstructure (e.g. the percentage of phases). More elaborate theoretical methods such as the asymptotic expansion method [3] and the convergence-based methods [4], [5], [6] allow to determine not only the homogenized constitutive law but also a way to construct the homogenized problem. In addition, convergence methods provide some quantities of interest (fields, differential operators and functionals). Results of these methods may be used to construct multiscale methods. A non-exhaustive list of these multiscale methods include the mean-field homogenization method [7], multiscale finite elements methods - MsFEM [8], the heterogeneous multiscale methods - HMM [9], [10], etc.

The application of such multiscale methods to study SMC is quite recent. In these methods, an elementary-cell problem is solved and the solution is used to compute the homogenized constitutive laws (electric and magnetic) [12], [13], [14]. The choice of the elementary cell is also crucial in order to

Manuscript received June, 24 2013; revised June, 24 2013. Corresponding author: I. Niyonzima (email: iniyonzima@doct.ulg.ac.be). accurately model real SMC structures. In this paper, we use an HMM method and exploit results from the asymptotic homogenization method and convergence-based theories in order to extend the computational homogenization method successfully used for modeling laminated ferromagnetic cores [10] to the case of SMC. Based on the HMM [9], it couples two types of problems: a macroscale problem that captures the slow variations of the overall solution, and many microscale problems that allow to determine the constitutive laws at the macroscale.

\section{Magnetodynamic Problem}

A magnetodynamic problem in a bounded domain $\Omega=\Omega_{c} \cup$ $\Omega_{c}^{C} \in \mathbb{R}^{3}$ is defined by the following Maxwell equations and constitutive laws [15]:

$$
\begin{array}{cc}
\operatorname{curl} \boldsymbol{h}=\boldsymbol{j}, & \operatorname{curl} \boldsymbol{e}=-\partial_{t} \boldsymbol{b}, \\
\boldsymbol{h}=\boldsymbol{H}(\boldsymbol{b}), & \boldsymbol{j}=\sigma \boldsymbol{e}+\boldsymbol{j}_{s},
\end{array}
$$

with $\boldsymbol{h}$ the magnetic field, $\boldsymbol{b}$ the magnetic flux density, $\boldsymbol{j}$ the electric current density, $\boldsymbol{j}_{s}$ the imposed electric current density (source) and $\boldsymbol{e}$ the electric field. The electric linear law involves $\sigma$, the (anisotropic) electric conductivity. The magnetic law $\boldsymbol{h}=\boldsymbol{H}(\boldsymbol{b})$ can be linear, nonlinear reversible or irreversible (i.e. with hysteresis) mapping. The domain $\Omega_{c}$ contains conductors whereas the domain $\Omega_{c}^{C}$ contains insulators and the inductor sources are in $\Omega_{s}$. Proper boundary conditions must also be imposed.

In this paper, we use the $\boldsymbol{a}-v$ formulation and write $\boldsymbol{b}$ and $\boldsymbol{e}$ as:

$$
\boldsymbol{b}=\operatorname{curl} \boldsymbol{a}, \quad \boldsymbol{e}=-\partial_{t} \boldsymbol{a}-\operatorname{grad} v,
$$

with $\boldsymbol{a}$ the magnetic vector potential and $v$ the electric scalar potential defined only in $\Omega_{c}$. The weak form of (1 a) reads [15]: find $\boldsymbol{a}$ and $v$ such that:

$$
\begin{array}{r}
\left(\boldsymbol{H}(\operatorname{curl} \boldsymbol{a}), \operatorname{curl} \boldsymbol{a}^{\prime}\right)_{\Omega}+\left(\sigma \partial_{t} \boldsymbol{a}, \boldsymbol{a}^{\prime}\right)_{\Omega_{c}}+\left(\sigma \operatorname{grad} v, \boldsymbol{a}^{\prime}\right)_{\Omega_{c}} \\
=-\left\langle\boldsymbol{n} \wedge \boldsymbol{h}, \boldsymbol{a}^{\prime}\right\rangle_{\Gamma_{h}}+\left(\boldsymbol{j}_{s}, \boldsymbol{a}^{\prime}\right)_{\Omega_{s}} \\
\left(\sigma \partial_{t} \boldsymbol{a}, \operatorname{grad} v^{\prime}\right)_{\Omega_{c}}+\left(\sigma \operatorname{grad} v, \operatorname{grad} v^{\prime}\right)_{\Omega_{c}}\left\langle\boldsymbol{n} \cdot \boldsymbol{j}, v^{\prime}\right\rangle_{\Gamma_{g}}
\end{array}
$$


and holds for all test functions $\boldsymbol{a}^{\prime}$ and $v^{\prime}$ in an appropriate function space. Surfaces $\Gamma_{h}$ and $\Gamma_{g}$ are the regions where natural boundary conditions on $\boldsymbol{h}$ and $\boldsymbol{j}$ are imposed, respectively. In the following, for the sake of conciseness, we omit these boundary terms.

\section{Multiscale MODEL}

We define the superscript $\varepsilon=l / L$ as the ratio between the finest scale $l$ and the scale of the material or the characteristic length of external loadings $L$.It denotes quantities with rapid spatial variations. The multiscale methods allow to reduce the huge computational cost resulting from the use of of classical numerical methods such as the FE method when solving Maxwell's system (1a-b) on a multiscale material.

The method, based on the scale separation assumption $(\varepsilon \ll$ 1), is illustrated in Fig. 1. A macroscale problem is defined on

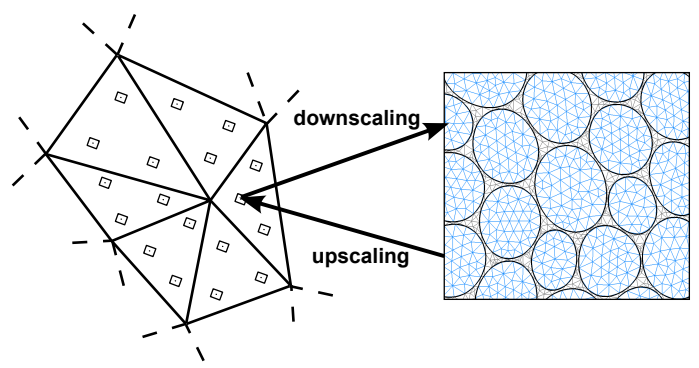

Figure 1. Scale transitions between macroscale (left) and mesoscale (right) problems. Downscaling (Macro to meso): obtaining proper boundary conditions and the source terms for the mesoscale problem from the macroscale solution. Upscaling (meso to Macro): effective quantities for the macroscale problem calculated from the mesoscale solution [16].

a coarse mesh covering the entire domain and many mesoscale problems are defined on small, finely meshed areas around some points of interest of the macroscale mesh (e.g. numerical quadrature points). Hereafter, the variables $\boldsymbol{x}$ and $\boldsymbol{y}$ are the macroscale and the mesoscale spatial positions, respectively and $\boldsymbol{y}=\boldsymbol{x}-\boldsymbol{x}_{b}$ is only defined on the cell domains with the origin at its barycenter $\boldsymbol{x}_{b}$. We define operators with respect to these variables. For instance, $\operatorname{curl}_{x}$ and $\operatorname{curl}_{y}$ denote curl operators with respect to $\boldsymbol{x}$ and $\boldsymbol{y}$. The subscripts $M, m$ and $c$ refer to the macroscale, the total and the correction mesoscale quantities, respectively. Only mesoscale quantities depend on the $\boldsymbol{y}$ coordinates. The average of $\boldsymbol{f}^{\varepsilon}$ over $\Omega_{m}$ (with $\left|\Omega_{m}\right|$ the volume of the cell) is denoted by

$$
<f^{\varepsilon}>_{\Omega_{m}}=\frac{1}{\left|\Omega_{m}\right|} \int_{\Omega_{m}} f^{\varepsilon} \mathrm{d} y .
$$

Further, we exploit the two-scale convergence theory [5] to determine the macroscale and mesoscale equations of a homogenized magnetodynamic problem. The theory holds if Maxwell's system (1a-b) has a solution for $\varepsilon \rightarrow 0$ and if the electromagnetic and differential operators are properly bounded [5]. It can be shown that the rapidly fluctuating fields $\boldsymbol{h}^{\varepsilon}(\boldsymbol{x}), \boldsymbol{b}^{\varepsilon}(\boldsymbol{x}), \boldsymbol{e}^{\varepsilon}(\boldsymbol{x})$ and $\boldsymbol{j}^{\varepsilon}(\boldsymbol{x})$ converge in the two-scale sense to some rapidly fluctuating two-scale limits $\boldsymbol{h}_{0}(\boldsymbol{x}, \boldsymbol{y}), \boldsymbol{b}_{0}(\boldsymbol{x}, \boldsymbol{y}), \boldsymbol{e}_{0}(\boldsymbol{x}, \boldsymbol{y})$ and $\boldsymbol{j}_{0}(\boldsymbol{x}, \boldsymbol{y})$ hence the name twoscale convergence. The purpose of the homogenization being to derive a macroscale model with slowly varying fields, a final step allows the passage from the two-scale limits to their averages $\boldsymbol{h}_{M}(\boldsymbol{x})=<\boldsymbol{h}_{0}(\boldsymbol{x}, \boldsymbol{y})>_{\Omega_{m}}, \boldsymbol{b}_{M}(\boldsymbol{x})=<\boldsymbol{b}_{0}(\boldsymbol{x}, \boldsymbol{y})>_{\Omega_{m}}$, $\boldsymbol{e}_{M}(\boldsymbol{x})=<\boldsymbol{e}_{0}(\boldsymbol{x}, \boldsymbol{y})>_{\Omega_{m}}$ and $\boldsymbol{j}_{M}(\boldsymbol{x})=<\boldsymbol{j}_{0}(\boldsymbol{x}, \boldsymbol{y})>_{\Omega_{m}}$. The differential operators curl $\boldsymbol{e}^{\varepsilon}$ and $\operatorname{curl} \boldsymbol{h}^{\varepsilon}$ also converge (in the two-scale sense) to some operators $\operatorname{curl}_{x} \boldsymbol{e}_{M}(\boldsymbol{x})+\operatorname{curl}_{y} \boldsymbol{e}_{c}(\boldsymbol{x}, \boldsymbol{y})$

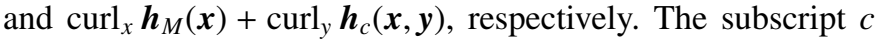
denotes the first correction term. Moreover, the fine-scale problem can be replaced by 1) a macroscale problem; 2) many mesoscale problems. The transfer of information between these problems and the weak formulations at both scales are detailed hereafter.

\section{A. Downscaling}

Mesoscale field quantities (subscript $m$ ) are governed by (1 a-b)-(2a-b) and $\boldsymbol{j}_{s} \equiv 0$. The two-scale convergence theory allows us to express the curl of the electric field at the mesoscale in terms of the electric field at the macroscale and a mesoscale correction, i.e. $\operatorname{curl}_{y} \boldsymbol{e}_{m}^{\varepsilon}=\operatorname{curl}_{x} \boldsymbol{e}_{M}+\operatorname{curl}_{y} \boldsymbol{e}_{c}$. Using the Faraday law at the macroscale together with the vector identity $\operatorname{curl}_{y}\left(\partial_{t} \boldsymbol{b}_{M} \wedge \boldsymbol{y}\right)=(n-1) \partial_{t} \boldsymbol{b}_{M}(n=2,3$ for $2 \mathrm{D}$ and $3 \mathrm{D}$ problems, respectively) we can write

$$
\operatorname{curl}_{y} \boldsymbol{e}_{m}^{\varepsilon}=\operatorname{curl}_{y}\left(\boldsymbol{e}_{c}+\boldsymbol{e}_{M}-\kappa\left(\partial_{t} \boldsymbol{b}_{M} \wedge \boldsymbol{y}\right)\right)
$$

with $\kappa=(n-1)^{-1}$, since $\operatorname{curl}_{y} \boldsymbol{e}_{M} \equiv 0$. This provides a natural development of $\boldsymbol{e}_{m}^{\varepsilon}$ in terms of a local, rapidly fluctuating component and a large scale component, for which we impose that $<\boldsymbol{e}_{m}^{\varepsilon}>_{\Omega_{m}}=\boldsymbol{e}_{M}$. We keep the multiscale form (i.e. we do not consider the two-scale homogenized form) for the Ampere equation $\operatorname{curl}_{y} \boldsymbol{h}_{m}^{\varepsilon}=\boldsymbol{j}_{0}$. We can thus use nonlinear and hysteretic magnetic laws $\boldsymbol{h}-\boldsymbol{b}$ for the mesoscale model. We develop $\boldsymbol{e}_{m}^{\varepsilon}$ and $\boldsymbol{b}_{m}^{\varepsilon}$ as follows:

$$
\left\{\begin{array}{l}
\boldsymbol{e}_{m}^{\varepsilon}=-\partial_{t} \boldsymbol{a}_{c}-\operatorname{grad}_{y} v_{c}+\boldsymbol{e}_{M}-\kappa\left(\partial_{t} \boldsymbol{b}_{M} \wedge \boldsymbol{y}\right) \\
\boldsymbol{b}_{m}^{\varepsilon}=\operatorname{curl}_{y} \boldsymbol{a}_{c}+\boldsymbol{b}_{M}
\end{array}\right.
$$

where the macroscale fields $\boldsymbol{b}_{M}$ and $\boldsymbol{e}_{M}$ are the source terms for the mesoscale problem. Boundary conditions for the mesoscale problem are also determined so as to respect the two-scale convergence of the physical fields: the convergence of the magnetic flux density $\boldsymbol{b}$ leads to a periodicity condition for the tangential component of the correction term of the magnetic vector potential $\boldsymbol{a}_{c}$, i.e.

$$
<\boldsymbol{b}_{m}^{\varepsilon}>_{\Omega_{m}}=\boldsymbol{b}_{M} \Longrightarrow \oint_{\Gamma_{m}} \boldsymbol{n} \wedge \boldsymbol{a}_{c} \mathrm{~d} y=0
$$

A condition of periodicity must also be imposed for the term $v_{c}$. The convergence of the electric field $\boldsymbol{e}$ leads to

$$
<\boldsymbol{e}_{m}^{\varepsilon}>_{\Omega_{m}}=\boldsymbol{e}_{M} \Longrightarrow \int_{\Omega_{m}} \boldsymbol{e}_{c} \mathrm{~d} y=0,
$$

for the correction term of the electric field $\boldsymbol{e}_{c}$.

\section{B. Upscaling}

The upscaling consists in computing the missing constitutive laws $\sigma_{M}, \boldsymbol{h}_{M}$ together with $\partial \boldsymbol{H}_{M} / \partial \boldsymbol{b}_{M}$ at the macroscale using the mesoscale fields. 
Thanks to the linearity of the electric law, the asymptotic expansion theory [3] can be applied. Therefore, we compute once for all the homogenized electric conductivity by solving a cell problem: find the periodic functions $\chi_{j}$ such that:

$$
\int_{\Omega_{m}}\left(\operatorname{grad}_{y} \psi^{\prime}\right)^{T} \sigma_{m}\left(\operatorname{grad}_{y} \chi_{j}-\boldsymbol{e}_{j}\right) \mathrm{d} y=0
$$

holds for all $\psi^{\prime}$ in an appropriate function space. The vector $\boldsymbol{e}_{j}$ is the unit vector in the $j^{\text {th }}$ spatial direction. The electric conductivity is then upscaled by means of:

$$
\left(\sigma_{M}\right)_{i j}=\left\langle\left(\sigma_{m}\right)_{i j}-\left(\sigma_{m}\right)_{i k} \frac{\partial \chi_{j}}{\partial y_{k}}\right\rangle_{\Omega_{m}} .
$$

The upscaling of the nonlinear magnetic law is performed by simple average as a consequence of the two-scale convergence of $\boldsymbol{h}^{\varepsilon}$ :

$$
<\boldsymbol{h}_{m}>_{\Omega_{m}}=\boldsymbol{h}_{M} .
$$

The tangent matrix $\partial \mathcal{H}_{M} / \partial \boldsymbol{b}_{M}$ for the Newton-Raphson scheme is obtained by finite differences [17].

\section{Weak formulations}

The macroscale formulation reads:

find $\boldsymbol{a}_{M}$ and $v_{M}$ such that

$$
\begin{aligned}
&\left(\boldsymbol{h}_{M}, \operatorname{curl}_{x} \underline{a}_{M}^{\prime}(\boldsymbol{x})\right)_{\Omega}+\left(\sigma_{M} \partial_{t} \boldsymbol{a}_{M}, \boldsymbol{a}_{M}^{\prime}\right)_{\Omega_{c}} \\
&+\left(\sigma_{M} \operatorname{grad}_{x} v_{M}, \boldsymbol{a}_{M}^{\prime}\right)_{\Omega_{c}}=\left(\boldsymbol{j}_{s}, \boldsymbol{a}_{M}^{\prime}\right)_{\Omega_{s}} \\
&\left(\sigma_{M} \partial_{t} \boldsymbol{a}_{M}, \operatorname{grad}_{x} v_{M}^{\prime}\right)_{\Omega_{c}}=-\left(\sigma_{M} \operatorname{grad}_{x} v_{M}, \operatorname{grad}_{x} v_{M}^{\prime}\right)_{\Omega_{c}}
\end{aligned}
$$

hold for all test functions $\boldsymbol{a}_{M}^{\prime}$ and $v_{M}^{\prime}$.

The mesoscale formulation reads:

find $\boldsymbol{a}_{c}$ and $v_{c}$ such that

$$
\begin{gathered}
\left(\boldsymbol{h}_{m}\left(\operatorname{curl}_{y} \boldsymbol{a}_{c}+\boldsymbol{b}_{M}\right), \operatorname{curl}_{y} \boldsymbol{a}_{m}^{\prime}\right)_{\Omega_{m}^{+}}+\left(\sigma_{m} \partial_{t} \boldsymbol{a}_{c}, \boldsymbol{a}_{m}^{\prime}\right)_{\Omega_{m c}^{+}} \\
\left(\sigma_{m} \operatorname{grad}_{y} v_{c}, \boldsymbol{a}_{m}^{\prime}\right)_{\Omega_{m c}}^{=}\left(\sigma_{m}\left(\boldsymbol{e}_{M}-\kappa \partial_{t} \boldsymbol{b}_{M} \wedge \boldsymbol{y}\right), \boldsymbol{a}_{m}^{\prime}\right)_{\Omega_{m c}} \\
\left(\sigma_{m} \partial_{t} \boldsymbol{a}_{c}, \operatorname{grad}_{y} v_{m}^{\prime}\right)_{\Omega_{m c}}^{+}\left(\sigma_{m} \operatorname{grad}_{y} v_{c}, \operatorname{grad}_{y} v_{m}^{\prime}\right)_{\Omega_{m c}=}^{=} \\
\left(\sigma_{m}\left(\boldsymbol{e}_{M}-\kappa \partial_{t} \boldsymbol{b}_{M} \wedge \boldsymbol{y}\right), \operatorname{grad}_{y} v_{m}^{\prime}\right)_{\Omega_{m c}^{+}}^{+}\left\langle\boldsymbol{n} \cdot \boldsymbol{j}_{M}, v_{m}^{\prime}\right\rangle_{\Gamma_{g m}}
\end{gathered}
$$

hold for all test functions $\boldsymbol{a}_{m}^{\prime}$ and $v_{m}^{\prime}$. Domains $\Omega_{m c}$ and $\Gamma_{g m}$ are the conducting part of the mesoscale domain and the boundary of $\Omega_{m c}$, respectively. The electric current density $\boldsymbol{j}_{M}=\sigma_{M} \boldsymbol{e}_{M}$ is obtained from the macroscale solution.

\section{Application}

A SMC material has been studied using a 2-D geometry with $40 \times 40$ square elementary cells surrounded by an inductor. Only a quarter of the geometry is modeled (Fig. 2-Left). Each cell comprises a conducting material surrounded by an insulating layer that represents the dielectric binder (Fig. 2Middle).

The insulation material is linear isotropic (with $\mu_{r}=1$ and $\sigma=0$ ). The conductor has an isotropic electric conductivity $\sigma=5 \mathrm{MS}$ and is governed by the following magnetic laws:

- nonlinear: $\mathcal{H}^{\varepsilon}\left(\boldsymbol{b}^{\varepsilon}\right)=\left(\alpha+\beta \exp \left(\gamma\left\|\boldsymbol{b}^{\varepsilon}\right\|^{2}\right)\right) \boldsymbol{b}^{\varepsilon}$ with $\alpha=388$, $\beta=0.3774$ and $\gamma=2.97$.

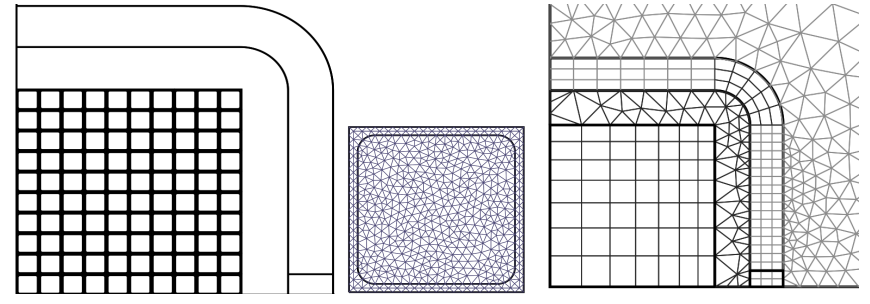

Figure 2. Left: geometry used for the validation of the model, taking advantage of symmetry. The source current is imposed in the surrounding inductor . Middle: mesh used for mesoscale computations. Right: mesh used for the macroscale problem.

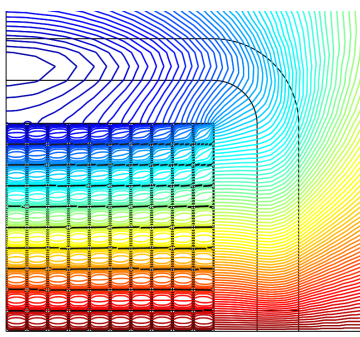

Figure 3. Nonlinear case - flux lines at $\mathrm{f}=50 \mathrm{KHz}$.

- Jiles-Atherton hysteresis model with parameters $m_{s}=$ $1,145,500 \mathrm{~A} / \mathrm{m}, a=59 \mathrm{~A} / \mathrm{m}, k=99 \mathrm{~A} / \mathrm{m}, c=0.55$ and $\alpha=1.3 \times 10^{-4}[18]$.

A sinusoidal electric current density with amplitude $\boldsymbol{j}_{s}=$ $3510^{8} \mathrm{~A} / \mathrm{m}^{2}$ and different frequencies is imposed. The reference solution is obtained by solving a FE problem on an extremely fine mesh (110986 elements)of the whole SMC structure. The mesoscale problems are solved on a square elementary cell meshed with 1278 elements (Fig. 2 - middle)).

The flux lines in Fig. 3 thanks to the isotropy of the material are smooth. The skin effect appears at the level of the cell.

The local $\boldsymbol{b} \boldsymbol{h}$ curve (Fig. 4) at $250 \mathrm{~Hz}$ shows a good agreement between the reference and the computational solutions. In (Fig. 5), cuts of the eddy currents $\boldsymbol{j}$ and the magnetic induction $\boldsymbol{b}$ are depicted. An excellent agreement is observed between the reference curve (labelled "Ref") and the local mesoscale solutions (labelled "Meso $1, \mathrm{Meso}_{2}, \mathrm{Meso}_{3}$ and $\left.\mathrm{MesO}_{4} "\right)$.

The instantaneous Joule losses are represented in Fig. 6. They are well estimated both with a reversible nonlinear law

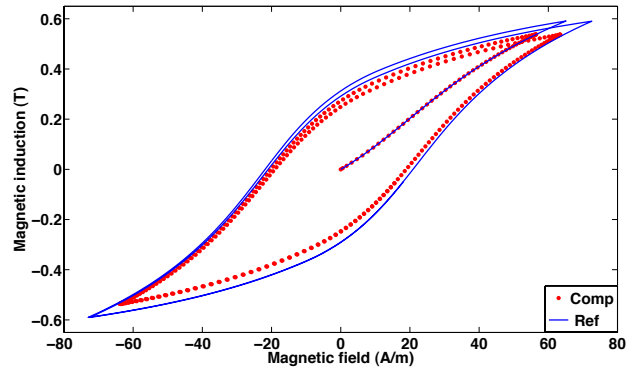

Figure 4. Reference (Ref) and computational (Comp) $\boldsymbol{h} \boldsymbol{b}$-hysteresis curves for a point located at $x_{1}=0.475 \mathrm{~mm}, x_{2}=0.475 \mathrm{~mm}(\mathrm{f}=250 \mathrm{~Hz})$. 

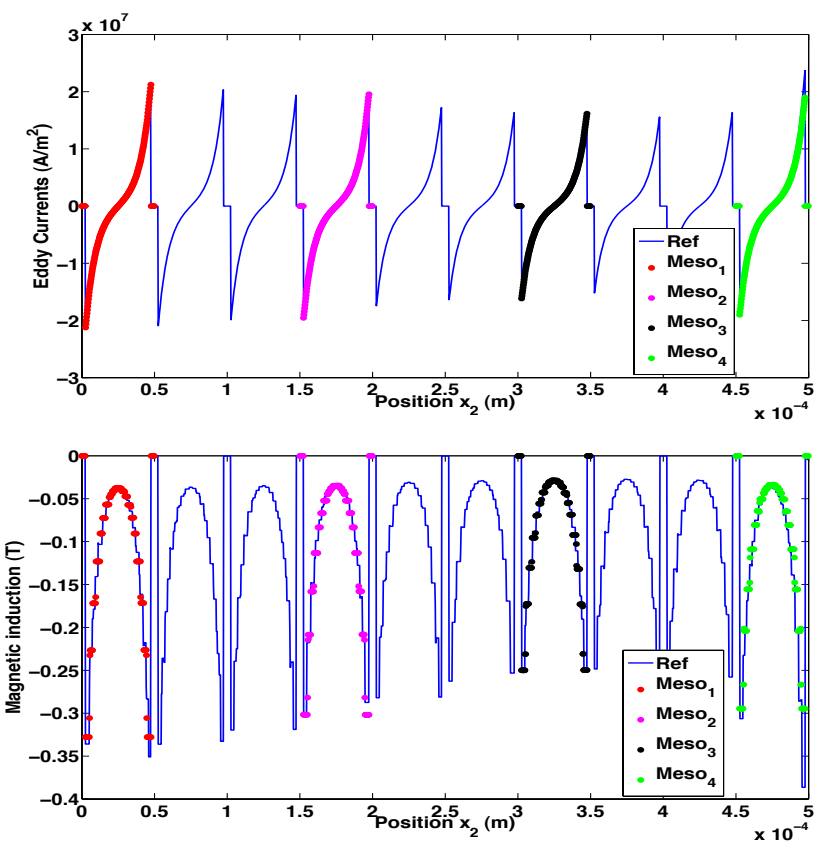

Figure 5. Nonlinear case - Top: Eddy currents $\boldsymbol{j}_{m}$ ( $x_{3}$ component) for a cut at $x_{1}=0.475 \mathrm{~mm}\left(t=6.6 \times 10^{-7} \mathrm{~s}\right)$. Bottom: Magnetic induction $\boldsymbol{b}_{m}\left(x_{1}\right.$ component) for a cut at $x_{1}=0.475 \mathrm{~mm}\left(t=6.6 \times 10^{-7} \mathrm{~s}\right)$ at $\mathrm{f}=50 \mathrm{KHz}$.
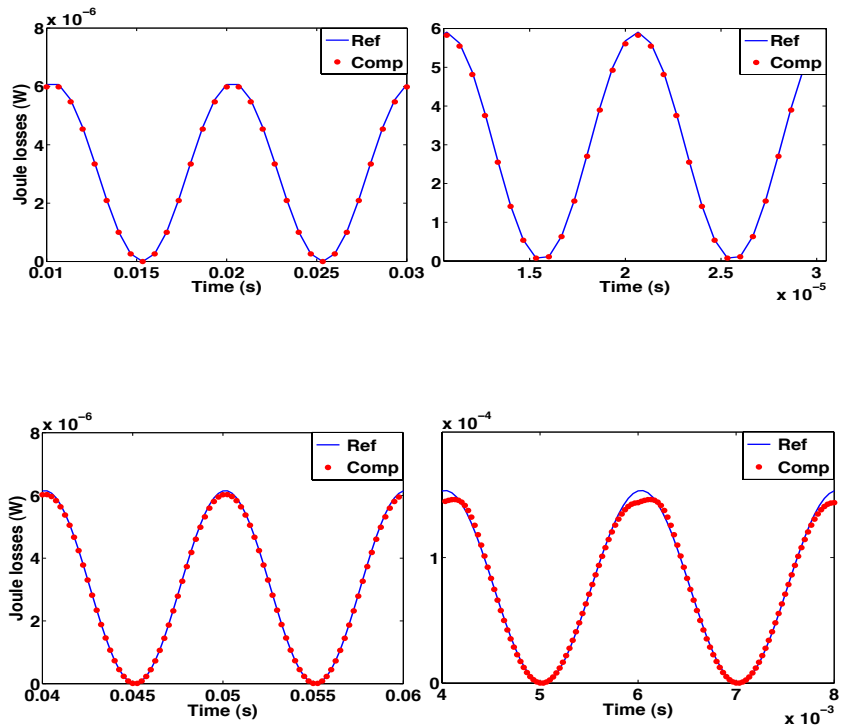

Figure 6. Instantaneous Joule losses. Nonlinear case - Top. Left: $\mathrm{f}=50 \mathrm{~Hz}$. Right: $\mathrm{f}=250 \mathrm{KHz}$. Hysteretic case - Top. Left: $\mathrm{f}=50 \mathrm{~Hz}$. Right: $\mathrm{f}=250 \mathrm{~Hz}$.

(top) and with hysteresis (bottom).

\section{ACKNOWLEDGMENT}

This work was supported by the Belgian French Community (ARC 09/14-02) and the Belgian Science Policy (IAP P7/02). Innocent Niyonzima is a fellowship beneficiary with the Belgian Research Training Fund for Industry and Agriculture (F.R.I.A.).

\section{REFERENCES}

[1] J. M. Garnett, "Colours in Metal Glasses and in Metallic Films," Philosophical Transactions of the Royal Society of London, Series A, vol. 203, pp385-420 1904.

[2] A. H. Sihvola, "Electromagnetic mixing formulas and applications," IEE Electromagnetic Waves Series, vol. 47, 1999.

[3] A. Bensoussan, J. L. Lions. and G. Papanicolau, "Asymptotic Analysis for Periodic Structures," American Mathematical Society, 2011.

[4] L. Tartar, "The General Theory of Homogenization: A Personalized Introduction," Springer, 2009.

[5] A. Visintin, "Homogenization of doubly-nonlinear equations," Rend. Lincei Mat. Appl., vol. 17, pp. 211-222, 2006.

[6] D. Cioranescu, A. Damlamian and G. Griso, "Eclatement périodique et homogénéisation," Comptes Rendus Mathématique, vol. 335, no. 1, pp. 99-104, 2002.

[7] R. Corcolle and L. Daniel, "Mean eld homogenization methods for piezoelectric composites," Progress In Electromagnetics Research Symposium, 2011.

[8] T. Y. Hou and X. H. Wu, "A multiscale finite element method for elliptic problems in composite materials and porous media," J.Comput. Phys. vol. 134, pp. 169-189, 1997.

[9] W. E, B. Engquist, X. Li, W. Ren and E. Vanden-Eijnden, "Heterogeneous multiscale methods: A review," Comm. Comp. Phys., vol. 2, no. 3, pp. 367-450, 2007

[10] I. Niyonzima, R. V. Sabariego, P. Dular and C. Geuzaine, "Computational homogenization for laminated ferromagnetic cores in magnetodynamics," IEEE Trans. Magn., vol. 49, no. 05, pp. 2049-2052, 2013.

[11] J. Gyselinck, R. V. Sabariego and P. Dular, "A Nonlinear time-domain homogenization technique for laminated iron cores in three-dimensional finite element models", IEEE Transactions on Magnetics, vol. 42, no. 4, pp. 763-766, 2006

[12] O. de la Barrière, O. Bottauscio, M. Chiampi and A. Manzin, "A multiscale approach to predict classical losses in soft magnetic composites," IEEE Trans. Magn., vol. 48, no. 4, 2012.

[13] O. Bottauscio, V. Chiado Piat, M. Chiampi, M. Codegone and A. Manzin, "Nonlinear homogenization technique for saturable soft magnetic composites," IEEE Trans. Magn., vol. 44, no. 11, 2008.

[14] C. Cyr, P. Viarouge, S. Clénet and J. Cros, "Methodology to study the influence of the microstructure of soft magnetic composites on their global magnetization curve," IEEE Trans. Magn., vol. 45, no. 03, 2009.

[15] A. Bossavit, "Computational Electromagnetism. Variational Formulations, Complementarity, Edge Elements," Academic Press, 1998.

[16] I. Niyonzima, R. V. Sabariego, P. Dular and C. Geuzaine, Finite element computational homogenization of nonlinear multiscale materials in magnetostatics, IEEE Transactions on Magnetics, vol. 48, no. 2, pp. 587-590, 2012.

[17] C. Miehe, Numerical Computational of Algorithmic (Consistent) Tangent Moduli in Large-Strain Computational Inelasticity, Computer Methods in Applied Mechanics and Engineering, vol. 134, pp. 223-240, 1996.

[18] A. Benabou, S. Clnet and F. Piriou, Comparison of Preisach and Jiles-Atherton models to take into account hysteresis phenomenon for finite element analysis, Journal of Magnetism and Magnetic Materials, vol. 261, pp. 305-310, 2003. 